\title{
Effectiveness of the first French psychoeducational program on unipolar depression: study protocol for a randomized controlled trial
}

\author{
Déborah Ducasse $e^{1,2,3^{*}}$, Philippe Courtet ${ }^{1,2,3}$, Maude Sénèque ${ }^{1,2,3}$, Catherine Genty ${ }^{1,2,3}$, Marie-Christine Picot ${ }^{4}$, \\ Raymund Schwan ${ }^{5}$ and Emilie Olié S,2,3 $^{1,2,3}$
}

\begin{abstract}
Background: Major Depressive Disorder (MDD) is highly prevalent and was associated with greater morbidity, mortality (including suicide), and healthcare costs. By 2030, MDD will become the leading cause of disability in high-income countries. Notably, among patients with a previous experience of a major depressive episode, it was indeed estimated that up to $85 \%$ of those patients will suffer from relapse. Two main factors were associated with a significantly higher risk of relapse: poor medication adherence and low self-efficacy in disease management. Interestingly, these issues could become the targets of psychoeducational programs for chronic diseases. Indded psychoeducational program for depression are recommended in international guidelines, but have not yet been proposed in France.

Methods/Design: We propose to evaluate the first French psychoeducational program for depression "ENVIE" in a multicenter randomized controlled trial. The group intervention will include 9 weekly sessions. Its aim is to educate patients on the latest knowledge on depression and effective treatments through didactic and interactive sessions. Patients will experiment the latest innovating psychological skills (from acceptance and commitment therapy) to cope with depressive symptoms and maintain motivation in behavioral activation. In total, 332 unipolar non-chronic (<2 years) outpatients with moderate to severe depression, without psychotic features, will be randomly allocated to the add-on ENVIE program $(N=166)$ or to a waiting list $(N=166)$. The follow-up will last 15 months and include 5 assessment visits. The primary endpoint will be the remission rate of the index episode at 15 months post-inclusion, defined by a Montgomery and Asberg Depression Rating Scale (MADRS) score $\leq 12$ over an 8-week period, and without relapse during follow-up. We will also assess the response rate and relapse at 15 months post-inclusion, hospitalization rate and adherence to treatment during the follow-up period, quality of life and global functioning upon inclusion and at 9 and 15 months post inclusion.
\end{abstract}

Discussion: If the proposed trial shows the effectiveness of the intervention, but also an increased remission rate in depressed outpatients at 15-months post-inclusion, in addition to improved treatment adherence in patients, it will further promotes arguments in favor of a wide dissemination of psychoeducational programs for depression.

Trial registration: This trial is registered under number 2015-A00249-40 (PURE clinical trial: NCT02501226) (June 30th, 2015).

Keywords: Psychoeducational program, Depression, Treatment adherence, Remission

\footnotetext{
* Correspondence: d-ducasse@chu-montpellier.fr

'Department of Emergency Psychiatry and Post Acute Care, CHU Montpellier, Hôpital Lapeyronie, 371 Avenue Gaston Giraud, 34295 Montpellier, France ${ }^{2}$ Inserm, U1061 Montpellier, University of Montpellier, Montpellier, France Full list of author information is available at the end of the article
} 


\section{Background}

MDD is associated with a considerable burden on individuals and societies and a lifetime prevalence of $12 \%$ in men and $26 \%$ in women [1], MDD ranks as the fourth disorder having the highest disease burden, accounting for $40.5 \%$ of DALYs caused by mental disorders [2]. MDD will be the leading cause of disability in highincome countries by 2030. MDD has been associated with greater morbidity, mortality (including suicide), healthcare utilization and costs [3]. Economic costs of depression have doubled in the past ten years, mainly due to an increase in indirect costs from loss of productivity $[4,5]$. There is a clear need to improve MDD management without increasing public mental health costs.

Although clinical improvement is often achieved in $60-70 \%$ of depressed patients treated with antidepressants, only about $35 \%$ will reach remission [6, 7]. In addition to antidepressants, complementary therapeutic strategies are needed. Furthermore, among patients who experienced one major depressive episode, it is estimated that up to $85 \%$ of them will relapse [1]. Moreover, patients who experienced two episodes, have a 70-90\% risk of having a third episode [1] The probability of subsequent episodes increases with the number of previous episodes, with a shorter interval between recurrences [8]. These data highlight the need to improve preventive actions to limit depression recurrence. Two main factors have been associated with a significantly higher risk of recurrence: poor medication adherence and low selfefficacy to manage one's illness (i.e. the patient's confidence in his or her ability to engage in behaviors to manage and prevent further recurrences) [1]. Interestingly, these two factors are targets of psychoeducational programs for chronic diseases. Indeed, these types of programs are designed to educate patients on topics that are essential and can empower them to become active in their recovery process through knowledge and information. Benefits of empowerment include improved selfimage, self-efficacy, as well as increased ability to cope with daily life and a higher likelihood of reaching treatment goals [9].

Even if most research on psychoeducation for patients with mood disorders was conducted in bipolar disorder [10], international clinical practice guidelines recently recommended psychoeducational interventions in MDD [11-13]. Psychoeducation in MDD was shown to: 1) reduce depressive symptoms [5, 14-29], 2) reduce the risk of relapse or recurrence [26, 30-33], 3) improve treatment adherence $[17,18,34], 4)$ increase quality of life [29] and global functionning [25, 35]. In these studies, the program "Coping with depression" (CWD) was the most used. CWD is a cognitive behavioral psychoeducative intervention based on the theory of depression and social learning [36], which aims at improving self- esteem, social skills, and management of depressive thoughts (skills in restructuring negative cognition). A recent meta-analysis [26] that included 25 randomized controlled trials showed that CWD was effective in reducing depressive symptoms (overall mean effect size of 0.28 (95\% CI:0.18-0.38)), and in preventing relapses (overall mean effect size of 0.62 (95\% CI: 0.43-0.91)). But CWD is complex and requires up to 16 sessions. Thus, other psychoeducational formats have been developed: psychoeducational cognitive workshops [14, 15, 19] and videotaped educational materials [37, 38]. However, most studies had a short-term follow-up limiting the interpretation of the effectiveness of these psychoeducational programs on the long-term management of MDD.

To our knowledge, no psychoeducational program for MDD is available in the French language. Thus, even though international guidelines recommend integrating a psychoeducational program in MDD treatment, none has yet been made available in France. We have developed the first French psychoeducational program for depression called "ENVIE". This group intervention consists of 9 weekly sessions. It aims at teaching patients the latest knowledge on depression and effective treatments, through didactic and interactive sessions. Patients will experiment the last innovating psychological skills to cope with depressive symptoms and maintain their motivation in behavioral activation [39, 40]. Moreover, the latest innovating psychological skills, which will be taught in the "ENVIE" program, belong to Acceptance and Commitment Therapy (ACT) [41]. Interestingly, ACT has shown equal to superior effectiveness compared to traditional cognitive behavioral therapy on unipolar depression [42-45]. Additionally, the specific impact of ACT skills was recently reported on suicidal risk $[46,47]$, which was highly associated with depression. Finally, patients will become experts and actors in the management of their disease in order to enhance treatment adherence, since lack of the latter has been strongly associated with an increased risk or relapse.

\section{Objective and research questions}

Primary objective:

The main objective is to compare the effectiveness (rate of remission of index episode at 15 months post inclusion, without relapse during follow-up) of the add-on ENVIE psychoeducational program vs. regular treatment only, in unipolar non-chronic ( $<2$ years) outpatients with moderate to severe depression, without psychotic features.

Secondary objectives:

Comparing add-on psychoeducational program with treatment as usual in regards to: 
- Decrease in depression intensity between inclusion and at 3, 6, 9 and 15 months post inclusion;

- Response rate 15 months after inclusion;

- Relapse rate 15 months after inclusion;

- Hospitalization rate during the follow-up period;

- Treatment adherence (antidepressants) between inclusion and at 3, 6, 9 and 15 months after inclusion;

- Changes in quality of life and global functioning between inclusion and at 9 and 15 months after inclusion;

- Modification of psychotropic treatments between inclusion and at 15 months post inclusion.

\section{Methods}

\section{Design}

We will conduct a two-arm randomized controlled trial, in order to compare the effectiveness of add-on psychoeducational program for unipolar depression vs. treatment as usual in depressed outpatients. This trial will take place in 11 French centers.

Based on medical records, eligible unipolar outpatients suffering from non-chronic (duration of current episode $<2$ years) major depressive episode (DSM-IV criteria) of moderate to severe intensity (MADRS score $>24$ ) will first be screened in each center. During the inclusion visit, participants matching all inclusion criteria and presenting no exclusion criteria will be informed by the investigator and invited to sign a consent form. They will be randomized in one of the two arms: add-on psychoeducational program (interventional group) or waiting list (control group). An independent researcher, not otherwise involved in the study, will perform the allocation. Each patient will be followed during 15 months with 5 visits: at baseline (V1), 3 months (V2), 6 months (V3), 9 months (V4) and 15 months (V5) after baseline. Blinded and trained evaluators will assess patients during the follow-up.

This research, involving human subjects, has been performed in accordance with the Declaration of Helsinki. The study protocol has been approved by an appropriate ethics committee (CPP Sud Méditerranée IV) (covering all 11 participating centres) and is registered under number 2015-A00249-40 (PURE clinical trial: NCT02501226) (June 30th, 2015).

\section{Sample size}

For the control group the full remission rate at 15 months is expected to be $30 \%$ [48]. If, in the experimental group, this rate is $15 \%$ higher, with a power of 0.80 and a two-tailed significance level at 0.05 , the sample size ends up to 166 patients per group. Because loss to follow-up will be considered as "not in remission", no adjusted sample size for taking dropouts into account has to be established. Thus, a total number of 332 subjects will be enrolled in the study. Each center will recruit between 20 and 40 patients: one to 2 psychoeducational groups of $8-10$ patients and 10-20 patients in the control group.

\section{Participants and procedure Inclusion and exclusion criteria}

We will include outpatients [1] aged 18-65 years [2] with a main diagnosis of non-psychotic non-chronic ( $<2$ years) major depressive episode (DSM-IV criteria) of moderate to severe intensity (Montgomery Asberg Depression Scale score >24) [49] taking at least one antidepressant [4] able to speak, read and understand French [5] and able to give written informed consent.

We will exclude subjects [1] with a current diagnosis of substance abuse or dependence in the 6 months prior to inclusion, excluding tobacco, [2] current psychotic features, [49] duration of current depressive episode $>2$ years, [4] current organic mental disorder or mental retardation, or severe comorbid medical condition, [5] lifetime history of schizophrenia, or schizoaffective or bipolar disorder, manic, hypomanic, or mixed episodes according to DSM-IV criteria, [6] sensory or cognitive disabilities, [7] hospitalized fulltime at the time of inclusion, [8] having a relationship or being employed by the sponsor or investigator. We will also exclude patients who are planning a long stay outside the region preventing compliance with the scheduled visits, and subjects participating in another trial.

\section{Recruitment}

Participants will be recruited via medical consultations in the 11 French investigation centers.

Written informed consent will be obtained from participants.

\section{Eligibility assessment and randomization}

People who apply for participation in the study will receive an information letter with comprehensive details on study procedures. Signed written informed consent will be obtained from all participants. They will be informed that they can withdraw from the intervention and/or study at any time without any negative consequences.

The first visit will consist in evaluating the following data:

Sociodemographic characteristics: age, gender, nationality, marital status, educational level, employment status;

$\bigcirc$ Psychopathology using the Mini-International Neuropsychiatric Interview (M.I.N.I.) [50]; 
Number of previous depressive episodes, date of onset of the current episode;

$\bigcirc$ Depression intensity using the Montgomery Asberg Depression Scale (MADRS) and Beck Depression Inventory (BDI);

$\bigcirc$ Global functioning using the Functioning

Assessment Short Test (FAST);

$\bigcirc$ Quality of life using the World Health Organization

Quality Of Life measure-26 (WHOQOL-26) scale;

$\bigcirc$ History of psychotropic treatment (name, dose);

$O$ Treatment adherence using the Medication

Adherence Rating Scale (MARS);

$\bigcirc$ Lifetime suicidality using the Columbia-Suicide

Severity Rating Scale (C-SSRS).

All inclusion and non-inclusion criteria will be checked by the study investigator. Patients will then be informed of their eligibility and randomized with the Capture Software System (Clinsight). Randomization will be performed with a permuted-block 1:1 ratio randomization list with varying block sizes and will be stratified in participating centers. Allocation concealment will be achieved by a centralized randomization procedure via an electronic case-report form.

\section{Assessments}

Patients will be assessed by clinicians blinded to treatment allocation, at inclusion (1-3 weeks before the intervention: visit 1) and at 3 months (end of the psychoeducational program: visit 2), 6 months (visit 3), 9 months (visit 4) and 15 months (visit 5) after inclusion. Patients will be advised to refrain from telling which group they were allocated to during visits.

See Fig. 1 for a detailed overview.

Investigators should make every effort to minimize the number of patients lost to follow-up and obtain a maximum of information on patients lost to follow-up, particularly in looking for any adverse events.

To limit patients lost to the touch, participants in the intervention group will be sent a text message on their mobile phone (internet-based free site) one day before each psychoeducational session. Each participant will receive two text messages: one the week before and the other the day prior to each follow-up visit.

\section{Intervention}

ENVIE: the first French psychoeducational group program Add-on psychoeducational group program

The intervention will consist of 9 weekly, 90-min sessions led by two trained educators (nurse and medical doctor). Each group will consist of 8-10 participants. The ENVIE program will provide participants with the latest medical knowledge on depression and effective treatments in a didactic manner [51-57], as well as the latest innovating psychological skills to cope with depressive symptoms and maintain motivation in behavioral activation [39, 40, 58]. These psychological skills belong to Acceptance and Commitment Therapy [58].

The program includes 9 sessions, each focusing on a specific theme or skill:

1. Presentation of the program and the matrix, and psychotherapeutic tools used during the entire program to motivate patients to behave in a manner that is consistent with what is important in their life [58].

2. Functional analysis of depression using the matrix (patients are taught to understand which symptoms/ behaviors are linked to depression, why they feel they are struggling, and what they can do to stop the struggle)

3. Causes leading to depression

4. Consequences of depression and comorbidities

5. Neurobiological bases of depression

6. Self-analysis of mood state, recognition of prodromal symptoms of relapse

7. Information about antidepressants, and evidencebased medical treatments for unipolar depression

8. Lifestyle recommendations

9. Behavioral activation skills

During sessions, participants will be encouraged to raise questions about anything they want to know about depression. To enhance the active role of patients, each session will be followed with take-home homework.

One or two psychoeducational groups of 8 to 10 patients will be planned in each center.

\section{Treatment as usual}

Treatment as usual will consist of the usual clinical management of depression including psychiatric evaluation and adaptation of pharmacological treatment. There will be no treatment restriction during the study period. The current pharmacological treatment will be recorded at each visit.

\section{Endpoints \\ Safety endpoints}

Incidence, relatedness, and severity of treatmentemergent adverse events will be evaluated at each visit until the end of study. Safety parameters assessed in this clinical trial will be recorded and evaluated through repeated clinical examinations of the patients. It relies on assessment of suicidality using the Columbia-Suicide Severity Rating Scale (C-SSRS) [59]: rate of suicidal behaviors (completed suicide and suicide attempts) at 15 months post inclusion, 


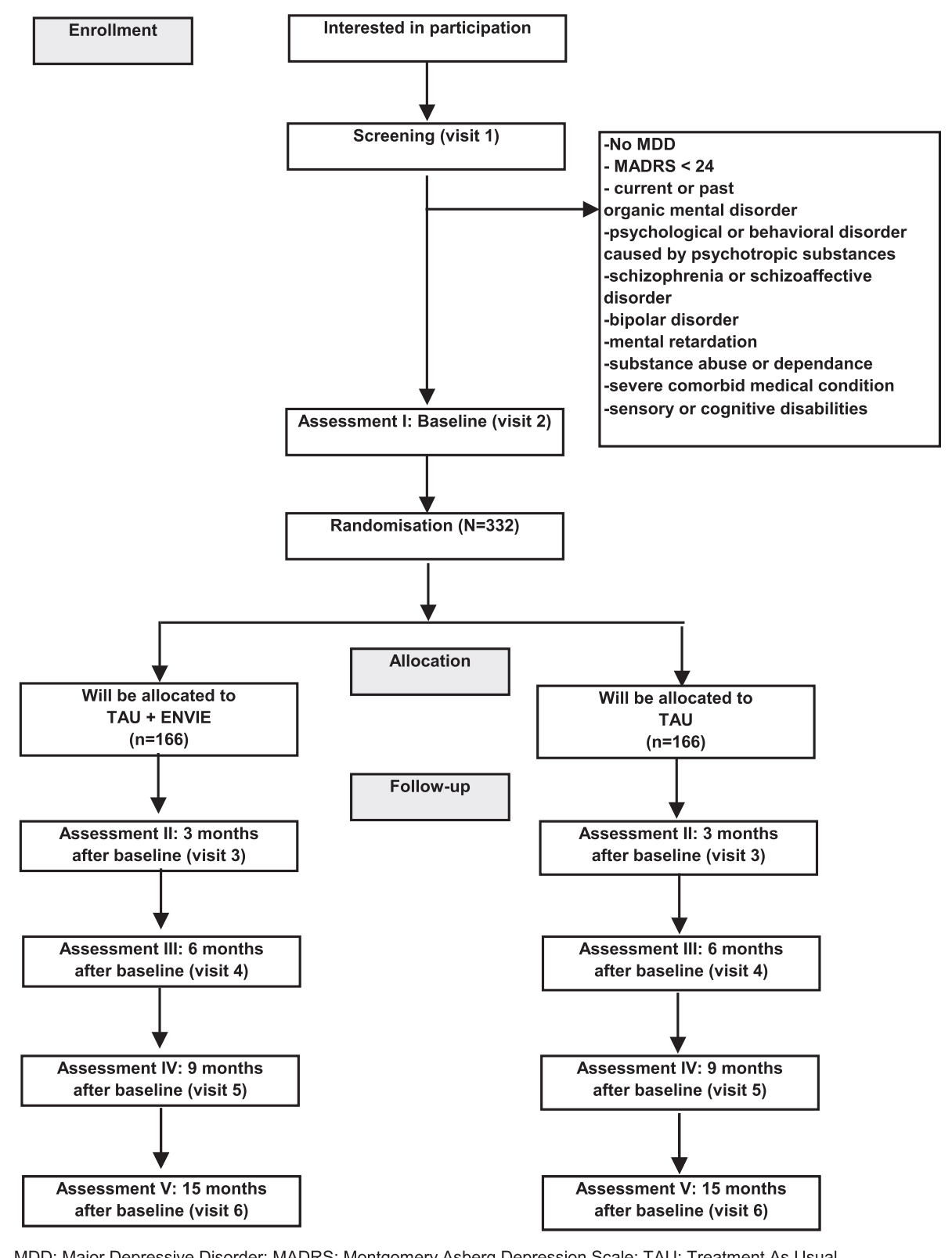

MDD: Major Depressive Disorder; MADRS: Montgomery Asberg Depression Scale; TAU: Treatment As Usual

Fig. 1 Study flow

variation of severity and intensity of suicidal ideations subscores between baseline and at 3, 6, 9 and 15 months after inclusion. If a patient exhibits a highrisk of suicide (increased suicide ideations, planning a suicide attempt) during a visit, investigators will contact the psychiatrist in charge of this patient.

Moreover, acceptability will be assessed. It will consist in the number of missed sessions, and satisfaction at 3 months post inclusion using a Likert scale ranging from 0 (not useful at all) to 10 (extremely useful) for the intervention group.

\section{Efficacy endpoints} Primary endpoint

Remission rate of the index episode at 15 months after inclusion, defined by a Montgomery and Asberg Depression Rating Scale (MADRS) [60] score $\leq 12$ for over 8weeks, without relapse during the follow-up period.

\section{Secondary endpoints}

- Variation of the depression intensity between baseline, and at 3, 6, 9 and 15 months after inclusion 
using MADRS and Beck Depression Inventory (BDI) [60, 61];

- Evolution of MADRS and BDI scores during follow-up;

- Response rate (50\% decrease in MADRS score) at 15 months after inclusion;

- Relapse rate (MADRS >12 after remission of the index episode) at 15 months after inclusion;

- Hospitalization rate during follow-up;

- Variation of global functioning, using the Functioning Assessment Short Test (FAST) [62], between baseline, and at 9 and 15 months after inclusion;

- Variation of quality of life using the World Health Organization Quality Of Life measure-26 (WHOQOL-26) scale [63] between baseline, and at 9 and 15 months after inclusion;

- Variation of treatment adherence using the Medication Adherence Rating Scale (MARS) [64] between baseline and 15 months after inclusion;

- Variation of benzodiazepines doses between baseline and at15 months after inclusion;

- Discontinuation rate of the antidepressant treatment at 15 months after inclusion.

\section{Statistical analysis}

\section{General statistical considerations}

All statistical analyses will be performed using SAS (SAS Institute, Cary, NC, USA).

For efficacy variables, we will use a Full Analysis Set, which will include all randomized subjects with a valid primary efficacy baseline measurement and at least one valid primary efficacy measurement. A robustness evaluation using the Completer Set (CS) will also be performed for the primary efficacy variable. The CS is a subset of the FAS and will include all subjects with a valid measurement at the end of the study Period (15 months) for the primary efficacy variable.

A summary of demographic data and baseline characteristics, as well as medical and procedure history will be presented for the whole population (all treatment groups combined) and for each treatment group. For continuous variables, summary statistics (n [number of available measurements], arithmetic mean, standard deviation [SD], median, interquartiles, minimum, and maximum) will be tabulated. For descriptive statistics of continuous variables, change from baseline and each time point will be displayed.

Frequency tables (frequency counts and percentages) will be presented for categorical data.

\section{Primary outcome}

The outcome of interest is the probability of remission achievement (rate of remission) of the index episode at
15 months post inclusion. Time to remission is defined as the number of days between the date of inclusion and remission or the last follow-up visit in case of no remission (maximum duration: 15 months). But the exact time of this event will not be known (the event could occur during the interval of two visits), so we will use methods for the analysis of interval-censored data. For calculation, all patient lost to follow-up will be considered as not in remission. A Cox proportional hazard model will be used to assess the impact of the intervention (Effect size). Covariates could be added in the Cox's proportional hazards regression model if both groups cannot be compared on baseline characteristics alone. The adjusted hazard ratios and their $95 \%$ confidence interval will be reported.

\section{Secondary outcomes}

The rate of response (decrease of MADRS score by $50 \%$ ), and rate of relapse (MADRS >12 after remission of the index episode) at 15 months after inclusion will be analyzed with the similar method used for the main criterion.

A mixed-model will be used to analyze:

1. Variations (intra- and between-group) of depression intensity using MADRS and BDI scores between inclusion, and at 3, 6, 9 and 15 months after inclusion.

2. And model the evolution of MADRS and BDI scores during follow-up

3. Variations (intra- and between-group) of global functioning (using the FAST) between inclusion, and at 9 and 15 months after inclusion,

4. Variations of quality of life using WHOQOL-26 scale between inclusion, and at 9 and 15 months after inclusion,

The rate of relapse (MADRS $>12$ after remission of the index episode) at 15 months after inclusion, the rate of hospitalization during follow-up and the discontinuation rate of antidepressant therapy at 15 months after inclusion will be analyzed using a chi-square test or Fisher's exact test if the chi-square is not valid.

The between-group comparison on variation in treatment adherence using MARS and changes in benzodiazepines doses between inclusion and 15 months post inclusion will be performed using the student- $t$ test if distribution is normal or the Mann-Whitney test, if otherwise.

\section{Safety analyses}

For the analysis of safety data (AEs, vital signs), RS will be used. Adverse events occurring during this study will be presented by system organ class (SOC), high level term (HLT), and preferred term [65] in a frequency table 
giving the number of events, number of subjects, and percentage of subjects who experienced the event by treatment group.

Subjects with multiple AEs will be counted only once within each PT, HLT, and SOC. Coding of the AEs will be performed with the Medical Dictionary for Regulatory Activities.

Vital signs variables and their changes from Baseline will be presented using descriptive statistics by visit and treatment group.

Rate of suicidal behaviors (completed suicide and suicide attempts) at 15 months after inclusion, variation of severity and intensity of suicidal ideations subscores between inclusion and at 3, 6, 9 and 15 months after inclusion will be reported for each group.

\section{Discussion}

Major Depressive Disorder is a highly prevalent disorder associated with a considerable loss of quality of life, increased mortality rates, and substantial economic costs. However, because of a lack of knowledge about this disease, many patients remain untreated although they need help. Also, among patients treated with adequate medication, only $35 \%$ will reach remission. Furthermore, the probability of MDD increases with the number of previous episodes, and the interval between recurrences does decrease with each new episode. However, two main factors associated with a significantly higher risk of relapse are: poor treatment adherence and low self-efficacy to manage depression, which are the primary targets in psychoeducation. Thus, psychoeducation has shown its effectiveness in reducing depressive symptoms and the risk of relapse or recurrence and in increasing patients' quality of life and global functioning. Moreover, psychoeducation is a simple add-on treatment, which can be delivered by any healthcare professional thus reducing costs and increasing the number of patients able to benefit from this program. Currently, few programs have been developed and those are mainly available in the English language.

This study will evaluate the effectiveness of the first French psychoeducational program associated with treatment as usual, via the rate of remission on depressed outpatients at 15 months of follow-up. A special emphasis will be given to teaching didactically the latest scientific knowledge about depression and effective treatments, and the latest innovating psychological skills to cope with depressive symptoms and maintain motivation in behavioral activation. Notably, psychological skills stem from Acceptance and Commitment Therapy, which is a brief integrative therapy (third wave behavior therapy, motivational interviewing, and existential therapy) with validated effectiveness on depression and suicidal risk. Finally, the
ENVIE program has already been conducted in the primary investigator centre, showing high adherence and satisfaction rates.

This study will have some limitations. First, as in most longitudinal studies we will need to deal with the problem of missing values. Thus, since loss to follow-up will be considered as "not in remission", positive results from this study will be particularly relevant. In order to limit patients lost to follow-up, participants in the intervention group will be sent a text message on their mobile phone one day before each psychoeducational session, and one week and one day before each follow-up visit. Second, patients will be randomized, but there is no double-blind, randomization since the patient will know if he/she belongs to the intervention or control group. Indeed, the control group will consist of a waiting list, and not a control group therapy. Third, we will not have any control over the treatment as usual in each of the two groups.

There will also be several strengths to this study, including the strong methodology of a randomized controlled design, validated assessments conducted by independent raters blinded to treatment condition, a sample defined by a standard diagnostic measure, an appropriate statistical analysis plan, and considering patients lost to follow-up as not in remission. Furthermore, the long-term follow-up (15 months), the assessment of remission and treatment adherence, and the latest innovative scientific didactic content of this short-duration program are highly relevant in these studies. Given these strengths, the results of the study should further enhance the evidence-based data supporting psychoeducational intervention for MDD. Finally, given the 11 French investigator centers implied in this study, the results could be easily generalized to depressed patients visiting French medical centers.

\section{Conclusion}

To overcome the gap between the need for treatment and evidence-based treatment availability and utilisation, cost-effective low-threshold interventions are strongly needed to be accessible to as many people as possible. Psychoeducation is one of them. In fact, a psychoeducational program seems the most available strategy to improve treatment adherence and selfefficacy to manage depression, two main factors associated with the risk of relapse. If the proposed trial shows not only the effectiveness of enhancing the rate of remission in depressed outpatients at 15 months follow-up but also improved treatment adherence in these patients, it would further strengthen the arguments for a wide dissemination of psychoeducational programs for depression. 


\section{Availability of data and materials} All data sets will be presented in additional supporting files.

\section{Competing interests}

The authors declare that they have no competing interests.

\section{Authors' contributions}

PC, RS, DD and EO participated in the figment of ENVIE program and the design of the study. SM and CG participated in the coordination between all the centers, and the legal and ethics formalities. MCP participated in the design of the study and will perform the statistical analysis. DD has written the present paper. All authors read and approved the final manuscript.

\section{Acknowledgements}

The authors thank B. Clement for the careful reading of the manuscript. The authors thank all investigators for their participation in this multicentric trial: Dr Bendimerad Patrick, Dr Bennabi Djamila, Dr Briki Malick, Dr Charpeaud Thomas, Dr Giordana Bruno, Dr Johnson Peter Kwaku, Dr Lerond Jérôme, Dr Nicolas Alain, Dr Nieto Isabel, Dr Verger Guillaume. The author thank Miss Emeline Massignan for her material assistance.

This project is funded by the National Clinical Hospital Research Program "PURE".

\section{Role of the funding source}

This study received financial support from French Health Ministry (Award Number: PHRC National 14-0214) and from CHU Montpellier (PHRC UF 9532). The study protocol has undergone peer-review by the funding body.

\section{Author details}

'Department of Emergency Psychiatry and Post Acute Care, CHU Montpellier, Hôpital Lapeyronie, 371 Avenue Gaston Giraud, 34295 Montpellier, France. İnserm, U1061 Montpellier, University of Montpellier, Montpellier, France. ${ }^{3}$ FondaMental Foundation, France. ${ }^{4}$ Clinical \& Epidemiological Reseach Unit, $\mathrm{CHU}$, Montpellier, France. ${ }^{5}$ Psychotherapeutic center of Nancy, CHU, Nancy, France.

\section{Received: 21 July 2015 Accepted: 29 October 2015}

Published online: 17 November 2015

\section{References}

1. Gopinath S, Katon WJ, Russo JE, Ludman EJ. Clinical factors associated with relapse in primary care patients with chronic or recurrent depression. J Affect Disord. 2007;101:57-63.

2. Whiteford HA, Degenhardt L, Rehm J, Baxter AJ, Ferrari AJ, Erskine HE, et al, Global burden of disease attributable to mental and substance use disorders: findings from the Global Burden of Disease Study 2010. Lancet. 2013;382:1575-86

3. Katon W, Lin E, Russo J, Unutzer J. Increased medical costs of a populationbased sample of depressed elderly patients. Arch Gen Psychiatry. 2003:60:897-903.

4. Smit F, Cuijpers P, Oostenbrink J, Batelaan N, de Graaf R, Beekman A. Excess costs of common mental disorders: population-based cohort study. J Ment Health Policy Econ. 2006;9:193-200.

5. Simon GE, Chisholm D, Treglia M, Bushnell D, Group L. Course of depression, health services costs, and work productivity in an international primary care study. Gen Hosp Psychiatry. 2002;24:328-35.

6. Simon GE, Von Korff M, Ludman EJ, Katon WJ, Rutter C, Unutzer J, et al. Cost-effectiveness of a program to prevent depression relapse in primary care. Med Care. 2002:40:941-50.

7. Trivedi MH, Rush AJ, Wisniewski SR, Nierenberg AA, Warden D, Ritz L, et al. Evaluation of outcomes with citalopram for depression using measurementbased care in STAR*D: implications for clinical practice. Am J Psychiatry. 2006;163:28-40.

8. Hardeveld F, Spijker J, De Graaf R, Nolen WA, Beekman AT. Prevalence and predictors of recurrence of major depressive disorder in the adult population. Acta Psychiatr Scand. 2010;122:184-91.

9. Linhorst DM, Hamilton G, Young E, Eckert A. Opportunities and barriers to empowering people with severe mental illness through participation in treatment planning. Soc Work. 2002;47:425-34.
10. Colom F. Keeping therapies simple: psychoeducation in the prevention of relapse in affective disorders. Br J Psychiatry. 2011;198:338-40.

11. Excellence NIfHC. NICE guidelines for Mental Health \& Behavioral conditions. 2010 [cited; Available from: http://guidance.nice.org.uk/Topic/Mental HealthBehavioural

12. Preventive Services Task Force. Screening for depression in adults: U.S. Preventive Services Task Force recommendation statement. Ann Intern Med. 2009:151:784-92

13. American Psychiatric Association. Practice guideline for the treatment of patients with major depressive disorder. 3rd ed. Arlington (VA): American Psychiatric Association (APA); 2010.

14. Scogin F, Jamison C, Gochneaur K. Comparative efficacy of cognitive and behavioral bibliotherapy for mildly and moderately depressed older adults. J Consult Clin Psychol. 1989;57:403-7.

15. Jamison C, Scogin F. The outcome of cognitive bibliotherapy with depressed adults. J Consult Clin Psychol. 1995;63:644-50.

16. Dowrick C, Dunn G, Ayuso-Mateos JL, Dalgard OS, Page H, Lehtinen V, et al. Problem solving treatment and group psychoeducation for depression: multicentre randomised controlled trial. Outcomes of Depression International Network (ODIN) Group. BMJ. 2000;321:1450-4.

17. Katon W, Rutter C, Ludman EJ, Von Korff M, Lin E, Simon G, et al. A randomized trial of relapse prevention of depression in primary care. Arch Gen Psychiatry. 2001;58:241-7.

18. Von Korff M, Katon W, Rutter C, Ludman E, Simon G, Lin E, et al. Effect on disability outcomes of a depression relapse prevention program. Psychosom Med. 2003;65:938-43.

19. Brown JS, Elliott SA, Boardman J, Ferns J, Morrison J. Meeting the unmet need for depression services with psycho-educational self-confidence workshops: preliminary report. Br J Psychiatry. 2004;185:511-5.

20. Clarke G, Eubanks D, Reid E, Kelleher C, O'Connor E, DeBar L. Overcoming depression on the Internet (ODIN) (2): a randomized trial of a self-help depression skills program with reminders. J Med Internet Res. 2005;7:2

21. Clarke G, Reid E, Eubanks D, O'Connor E, deBar L, Kelleher C. Overcoming depression on the Internet (ODIN): a randomised controlled trial of an Internet depression skills intervention program. J Med Internet Res. 2002;4:e14.

22. Christensen $\mathrm{H}$, Griffiths KM, Jorm AF. Delivering interventions for depression by using the internet: randomised controlled trial. BMJ. 2004;328:265.

23. Dalgard OS. A randomized controlled trial of a psychoeducational group program for unipolar depression in adults in Norway (NCT00319540). Clin Pract Epidemiol Ment Health. 2006;2:15.

24. Butler LD, Waelde LC, Hastings TA, Chen XH, Symons B, Marshall J, et al. Meditation with yoga, group therapy with hypnosis, and psychoeducation for long-term depressed mood: a randomized pilot trial. J Clin Psychol. 2008;64:806-20.

25. Hansson M, Bodlund O, Chotai J. Patient education and group counselling to improve the treatment of depression in primary care: a randomized controlled trial. J Affect Disord. 2008;105:235-40.

26. Cuijpers P, Munoz RF, Clarke GN, Lewinsohn PM. Psychoeducational treatment and prevention of depression: the "Coping with Depression" course thirty years later. Clin Psychol Rev. 2009;29:449-58.

27. Donker T, Griffiths KM, Cuijpers P, Christensen H. Psychoeducation for depression, anxiety and psychological distress: a meta-analysis. BMC Med. 2009;7:79.

28. Meyer B, Berger T, Caspar F, Beevers CG, Andersson G, Weiss M. Effectiveness of a novel integrative online treatment for depression (Deprexis): randomized controlled trial. J Med Internet Res. 2009;11:e15.

29. Casanas R, Catalan R, del Val JL, Real J, Valero S, Casas M. Effectiveness of a psycho-educational group program for major depression in primary care: a randomized controlled trial. BMC Psychiatry. 2012;12:230.

30. Shimazu K, Shimodera S, Mino Y, Nishida A, Kamimura N, Sawada K, et al. Family psychoeducation for major depression: randomised controlled trial. Br J Psychiatry. 2011;198:385-90.

31. Shimodera S, Furukawa TA, Mino Y, Shimazu K, Nishida A, Inoue S. Costeffectiveness of family psychoeducation to prevent relapse in major depression: results from a randomized controlled trial. BMC Psychiatry. 2012;12:40.

32. Morokuma I, Shimodera S, Fujita H, Hashizume H, Kamimura N, Kawamura A, et al. Psychoeducation for major depressive disorders: a randomised controlled trial. Psychiatry Res. 2013;210:134-9.

33. Stangier U, Hilling C, Heidenreich T, Risch AK, Barocka A, Schlosser R, et al. Maintenance cognitive-behavioral therapy and manualized 
psychoeducation in the treatment of recurrent depression: a multicenter prospective randomized controlled trial. Am J Psychiatry. 2013;170:624-32.

34. Seedat S, Haskis A, Stein DJ. Benefits of consumer psychoeducation: a pilot program in South Africa. Int J Psychiatry Med. 2008;38:31-42.

35. Cuijpers P. Bibliotherapy in unipolar depression: a meta-analysis. J Behav Ther Exp Psychiatry. 1997;28:139-47.

36. Teri L, Lewinsohn PM. Individual and group treatment of unipolar depression: comparison of treatment outcome and identification of predictors of successful treatment outcome. Behav Ther. 1986;17:215-28.

37. Simon GE, Katon WJ, VonKorff M, Unutzer J, Lin EH, Walker EA, et al. Costeffectiveness of a collaborative care program for primary care patients with persistent depression. Am J Psychiatry. 2001;158:1638-44.

38. Simon GE, Manning WG, Katzelnick DJ, Pearson SD, Henk HJ, Helstad CS. Cost-effectiveness of systematic depression treatment for high utilizers of general medical care. Arch Gen Psychiatry. 2001;58:181-7.

39. Hollon SD, Ponniah K. A review of empirically supported psychological therapies for mood disorders in adults. Depress Anxiety. 2010;27:891-932.

40. Bohlmeijer ET, Fledderus M, Rokx TA, Pieterse ME. Efficacy of an early intervention based on acceptance and commitment therapy for adults with depressive symptomatology: Evaluation in a randomized controlled trial. Behav Res Ther. 2011;49:62-7.

41. National action alliance for suicide prevention. www.suicide-researchagenda.org.

42. Forman EM, Herbert JD, Moitra E, Yeomans PD, Geller PA. A randomized controlled effectiveness trial of acceptance and commitment therapy and cognitive therapy for anxiety and depression. Behav Modif. 2007;31:772-99.

43. Zettle RD, Rains JC, Hayes SC. Processes of change in acceptance and commitment therapy and cognitive therapy for depression: a mediation reanalysis of Zettle and Rains. Behav Modif. 2011;35:265-83.

44. Zettle RD, Hayes SC. Dysfunctional control by client verbal behavior: the context of reason-giving. Anal Verbal Behav. 1986;4:30-8.

45. Zettle RD, Rains JC. Group cognitive and contextual therapies in treatment of depression. J Clin Psychol. 1989;45:436-45.

46. Luoma JB, Villatte JL. Mindfulness in the treatment of suicidal individuals. Cogn Behav Pract. 2012;19:265-76.

47. Ducasse D, René E, Béziat S, Guillaume S, Courtet P, Olie E. Acceptance and commitment therapy for management of suicidal patients: a pilot study. Psychother Psychosom. 2014: In Press.

48. Vuorilehto MS, Melartin TK, Isometsa ET. Course and outcome of depressive disorders in primary care: a prospective 18-month study. Psychol Med. 2009; 39:1697-707.

49. Pearson TA, Mensah GA, Alexander RW, Anderson JL, Cannon 3rd RO, Criqui $M$, et al. Markers of inflammation and cardiovascular disease: application to clinical and public health practice: A statement for healthcare professionals from the Centers for Disease Control and Prevention and the American Heart Association. Circulation. 2003;107:499-511.

50. Sheehan DV, Lecrubier Y, Sheehan KH, Amorim P, Janavs J, Weiller E. The Mini-International Neuropsychiatric Interview (M.I.N.I.): the development and validation of a structured diagnostic interview for DSM-IV and CIM-10. J Clin Psychiatry. 1998;59:22-3.

51. American Psychiatric Association. Diagnostic and statistical manual of mental disorders, fifth edition (DSM5). 2013.

52. Klengel T, Binder EB. Gene-environment interactions in major depressive disorder. Can J Psychiatry. 2013;58:76-83.

53. Kupfer DJ, Frank E, Phillips ML. Major depressive disorder: new clinical, neurobiological, and treatment perspectives. Lancet. 2012;379:1045-55.

54. Caspi A, Hariri AR, Holmes A, Uher R, Moffitt TE. Genetic sensitivity to the environment: the case of the serotonin transporter gene and its implications for studying complex diseases and traits. Am J Psychiatry. 2010; 167:509-27.

55. Bremner JD, Narayan M, Anderson ER, Staib LH, Miller HL, Charney DS. Hippocampal volume reduction in major depression. Am J Psychiatry. 2000; 157:115-8.

56. Bremner JD. Structural changes in the brain in depression and relationship to symptom recurrence. CNS Spectr. 2002;7:129-30. 35-9.

57. Stahl SM. Stahl's Essential Psychopharmacology: Neuroscientific Basis and Practical Applications Cambridge University Press. 4th ed. 2013.

58. Schoendorff B. La thérapie d'acceptation et d'engagement: guide clinique. Bruxelles: De Boeck ed; 2011.

59. Posner K, Brown GK, Stanley B, Brent DA, Yershova KV, Oquendo MA, et al. The Columbia-suicide severity rating scale: initial validity and internal consistency findings from three multisite studies with adolescents and adults. Am J Psychiatry. 2011;168:1266-77.

60. Montgomery SA, Asberg M. A new depression scale designed to be sensitive to change. Br J Psychiatry. 1979;134:382-9.

61. Beck AT. Beck Depression Inventory. 2nd ed. San Antonio: The Psychological Corporation ed; 1996.

62. Rosa AR, Sanchez-Moreno J, Martinez-Aran A, Salamero M, Torrent C, Reinares $M$, et al. Validity and reliability of the Functioning Assessment Short Test (FAST) in bipolar disorder. Clin Pract Epidemiol Ment Health. 2007;3:5.

63. Murphy B, Herman H, Hawthorne G, Pinzone T, Evert H. Australian WHOQoL instruments: User's manual and interpretation guide. Melbourne: Australian WHOQoL Field Study Centre ed; 2000

64. Thompson K, Kulkarni J, Sergejew AA. Reliability and validity of a new Medication Adherence Rating Scale (MARS) for the psychoses. Schizophr Res. 2000;42:241-7.

65. Hackett RA, Hamer M, Endrighi R, Brydon L, Steptoe A. Loneliness and stress-related inflammatory and neuroendocrine responses in older men and women. Psychoneuroendocrinology. 2012;37:1801-9.

\section{Submit your next manuscript to BioMed Central and take full advantage of:}

- Convenient online submission

- Thorough peer review

- No space constraints or color figure charges

- Immediate publication on acceptance

- Inclusion in PubMed, CAS, Scopus and Google Scholar

- Research which is freely available for redistribution

Submit your manuscript at www.biomedcentral.com/submit 